\title{
Celibato en el Judaísmo Antiguo ${ }^{1}$
}

\author{
Pieter W. VAN DER HORST \\ Utrecht University
}

«Creced y multiplicaos (peru u-revu)» (Gen.1:28). De acuerdo con la Biblia, éstas fueron las primeras palabras que Dios dirigió a los hombres. Si estas palabras primeras fueran un imperativo por el que la humanidad se viera obligada a producir una numerosa descendencia, ciertamente que con ellas no se crearía el clima ideal para el desarrollo del ascetismo sexual ${ }^{2}$. No puede sorprender, por tanto, que para el judaísmo, a diferencia de lo que ocurre con el cristianismo, el celibato nunca tuviera la condición de un ideal $^{3}$. El cristianismo -se dice con frecuencia-se separó del ideal bíblico y judío al dejarse influir por la filosofía platónica, con sus afirmaciones, frecuentemente negativas, de la materialidad y la corporeidad. Hace ya tiempo que se cuestiona, y con razón ${ }^{4}$, esta pintura en blanco y negro, por un lado, del judaísmo, que fiel a sus raíces bíblicas mantiene una actitud positiva hacia el cuerpo, y por otro del cristianismo, que se habría desviado de este camino, desarrollando una actitud negativa.

Por supuesto que, antes de comenzar a discutir el tema, sería aconsejable presentar una definición del ascetismo, pero aparte

\footnotetext{
${ }^{1}$ El autor desea expresar su agradecimiento a la profesora Carmen LÓPEZ ALONSO por su cuidada traducción de la versión inglesa del artículo.

${ }^{2}$ Para la historia de la recepción de este texto bíblico, véase J. COHEN, «Be Fertile and Increase, Fill the Earth and Master It». The Ancient and Medieval Career of a Biblical Text (Ithaca - London 1980).

${ }^{3}$ Cf. H. StRATHMANn, Geschichte der frühchristlichen Askese (Leipzig 1914) págs. 16-40 («Der unasketische Grundzug der palästinensisch-jüdischen Frömmigkeit»).

4 Véase, p.e., D. SATRAN, «Askese VI: Judentum», RGG I (1998) págs. 839-840.

Sefarad 62 (2002) págs. 85-98

(c) CSIC

ISSN 037-0894
} 
del hecho de que no exista una definición sobre la que todo el mundo esté de acuerdo ${ }^{5}$, he decidido restringir mi discusión a un solo aspecto del fenómeno del ascetismo, si bien el más llamativo: la abstinencia sexual. Otra de las restricciones es que no me referiré a la abstinencia sexual temporal, limitándome únicamente a la permanente. También caen fuera del alcance de esta pequeña contribución otros aspectos del ascetismo, como el ayuno, el autocastigo, la pobreza voluntaria, la anacoresis, etc. El período en consideración es el comprendido entre Alejandro Magno y Mahoma.

No cabe duda del hecho de que en la literatura rabínica está plenamente confirmado el ideal bíblico de fundar una familia. Son frecuentes los comentarios rabínicos en los que se desaprueba el estado de soltería ${ }^{6}$. Por poner un ejemplo: en el Talmud de Babilonia (Quiddushin 29b) se lee que si un hombre ha cumplido los veinte años sin haberse casado, transgrede un mandato divino y provoca la cólera de Dios. Aunque algunos están dispuestos a ampliar el límite de edad a los veinticuatro años, el sentimiento sigue siendo el mismo: uno tiene que fundar una familia a su debido tiempo y, si no lo hace, es realmente tan nocivo como alguien que derrama sangre, pues priva de vida a una descendencia potencial. Más aún, un hombre soltero por fuerza estará constantemente pensando en el sexo y, en consecuencia, se estará poniendo trabas para aspirar a fines más elevados. Esta es la posición del Talmud ${ }^{7}$.

Había excepciones, no obstante. Shim'on ben Azai era un famoso y respetado rabino (de principios del siglo II d.C.) que no se

\footnotetext{
5 S. D. FRAADE, «Ascetical Aspects of Ancient Judaism», en Jewish Spirituality from the Bible through the Middle Ages, ed. A. GREEN (London 1985) págs. 253-288: págs. 253-257. Fraade utiliza la siguiente definición de Vööbus: «Ascetismo, en religión, es la práctica de la negación de los deseos físicos o psicológicos con el fin de conseguir un ideal o un fin espiritual» (A. VÖÖBUS, en Encyclopaedia Británica, vol. 2 [15 ed. Chicago 1976] pág. 135).

${ }^{6}$ Véase G. F. MOORE, Judaism in the First Centuries of the Christian Era, the Age of the Tannaim, vol. 2 (Cambridge 1927, repr. 1966) págs. 119-120, para referencias. Sobre polémicas en fuentes judías no rabínicas contra los que permanecen solteros, véase P. W. VAN DER HORST, The Sentences of Pseudo-Phocylides (Leiden 1978) págs. 225-227.

${ }^{7}$ Más datos en D. M. Feldman, Birth Control in Jewish Law. Marital Relations, Contraception, and Abortion as Set Forth in the Classic Texts of Jewish Law (Westport $1980=$ New York 1968), págs. 46-80.
} 
casó jamás ${ }^{8}$. Sus colegas le criticaban continuamente por ello, pero él solía contestar: «¿Qué puedo hacer? ¡Mi alma tiene sed de la Torá! Dejad que otros se ocupen de que el mundo siga su curso» (Tosefta, Jebamot 8:7; Talmud Bab. Yebamot 63b). Con esto estaba diciendo que la humanidad no moriría si él permanecía soltero. «Mi alma tiene sed de la Torá» implica que estaba tan obsesionado con su estudio de la Torá, el ideal más elevado de la cultura rabínica, que no le quedaba espacio, ni tiempo, para una esposa. En la figura de Shim'on ben Azai nos encontramos con un rabino que, a pesar de Gen. 1:28 y de la presión social de sus correligionarios, renuncia voluntariamente al matrimonio y a fundar una familia para poder alcanzar un fin más elevado con mucha mayor determinación, es decir, un mayor conocimiento de la Torá $y$, por tanto, un mejor entendimiento de la voluntad de Dios 9.

Volvamos primero al período prerrabínico para ver si las fuentes judías tempranas, las del judaísmo del Segundo Templo, sugieren que ya entonces había judíos que permanecían solteros para perseguir un fin más elevado. Este es, ciertamente, el caso. Una notable figura ascética es la descrita por Flavio Josefo. En su autobiografía, escrita hacia el final del siglo I d.C., narra su búsqueda de guía espiritual, iniciada cuando tenía unos dieciséis años. Flavio Josefo se dirigió al «mercado» de los fariseos, al de los saduceos y al de los esenios, pero quedó insatisfecho con sus experiencias con estos grupos. Escribe entonces: «Cuando oí hablar de un tal Banus, que vivía en el desierto usando como vestido lo que le proporcionaban los árboles y como alimento lo que producía la tierra espontáneamente, que se bañaba varias veces, en agua fría, de día y de noche, para purificarse, me convertí en su discípulo. Viví con él tres años» (Vita 11$)^{10}$. Por el contexto está claro que sobre lo

\footnotetext{
${ }^{8}$ Véase Tosefta, Jebamot 8:7; también G. BADER, The Encyclopedia of Talmudic Sages (Northvale - London 1988) págs. 295-299, para un examen del material rabínico sobre Ben Azai.

${ }^{9}$ Véase la frase que inicia el capítulo de D. BOYARIN, «Lusting After Learning», en Carnal Israel: Reading Sex in Talmudic Culture (Berkeley 1993) pág. 134: «Las absolutas y contradictorias demandas de matrimonio y de dedicación al estudio de la Torá siguieron siendo una de las tensiones no resueltas dentro de la cultura rabínica».

${ }^{10}$ Versión española de M. RodríGuez de SEPúlveda (Madrid 1994). Véanse útiles comentarios en S. MASON, Life of Josephus. Translation and Commentary (Leiden 2001) págs. 18-20.
} 
que Josefo está escribiendo es sobre una búsqueda religiosa, y que por esa razón hemos de asumir, aunque Josefo no lo diga de forma explícita, que es por razones religiosas por las que Banus se retiró al desierto (lo que es, por cierto, una notable manifestación de lo que tres siglos después, y a una escala mucho más amplia, vuelve a producirse en los círculos cristianos, cuando los llamados padres del desierto se establecen en él) ${ }^{11}$. Podemos asumir sin riesgo que ešte hombre, vestido con cortezas de árbol, no deambulaba por el desierto con mujer e hijos; era, sin duda, intencionadamente célibe. Es un gran enigma saber lo que Josefo aprendió realmente en esos tres años con Banus, ya que, tras ese período de entrenamiento, a lo largo de su vida se casó ipor lo menos cuatro veces!

Es el mismo Josefo quien escribe extensamente y con aparente simpatía sobre los esenios, a los que describe como un grupo de filósofos célibes: «Esquivan los placeres como un vicio y miran la templanza y el control de las pasiones como una virtud especial. Desdeñan el matrimonio, pero adoptan a los hijos de otros hombres, cuando ya son flexibles y dóciles (...) y los moldean de acuerdo con sus propios principios» (Bellum 2:120) ${ }^{12}$. Al final de esta larga sección, que muestra la admiración de Josefo por este grupo, añade lo siguiente: «Hay otra orden de esenios que, aunque de acuerdo con el resto en su modo de vida, difiere de ellos en su visión sobre el matrimonio. Piensan que aquellos que renuncian a casarse rompen con la función principal de la vida, la propagación de la especie, y, todavía peor, si todos adoptaran el mismo punto de vista, la humanidad pronto desaparecería» (Bellum 2:160). De todos modos está claro que, de acuerdo con Josefo, había un considerable grupo de judíos que se negaban a casarse, de nuevo por razones religiosas. Sin embargo, no profundiza en las razones de ello ${ }^{13}$.

Filón, su contemporáneo de más edad, confirma esto al afirmar explícitamente, en su descripción de los esenios: «Ningún esenio

\footnotetext{
$"$ Véase la introducción de P. W. VAN DER HORST, De Woestijnvaders. Levensverhalen van kluizenaars uit her vroege christendom (Amsterdam 1998).

${ }^{12}$ Para una discusión amplia, véase T. S. BEALL, Josephus' Description of the Essenes Illustrated by the Dead Sea Scrolls (Cambridge 1988) págs. 38-41.

${ }^{13}$ Muchos años después Josefo repite esta información en su Antiquitates Judaicae 18:21.
} 
toma nunca mujer» (Hypothetica $11: 14$ ) ${ }^{14}$, pero añade que esto se debe a que las mujeres son criaturas egoístas, excesivamente celosas y maestras en corromper la moral de sus maridos y en seducirlos con sus continuas imposturas, etc. Filón apenas puede contenerse al presentar esta pintura tan negativa de las mujeres, pero es cuestionable si estas razones fueron realmente los motivos por los que los esenios no se casaban. Posiblemente fueran las razones por las que el propio Filón considerara bueno para él no casarse, pero desafortunadamente no podemos sustanciar esta opinión.

No obstante, esto nos lleva a la cuestión de si el movimiento esenio era, completa o parcialmente, célibe. Se ha afirmado con frecuencia que lo que Filón y Josefo presentan son descripciones no históricas, que se han visto muy influidas por las ideas helenizantes o por los ideales helenísticos ${ }^{15}$. Pero ¿es realmente así? Caben pocas dudas de que la descripción de Filón sobre los esenios rebosa de terminología filosófica griega, que también le gusta utilizar en otros escritos. Pero ésta podría haber sido, así mismo, su manera de presentar unos datos que, en ultima instancia, están basados en hechos históricos. Veamos esto con mayor detenimiento ${ }^{16}$. Para empezar podemos afirmar que, independientemente de Filón y de Josefo, el enciclopédico erudito romano Plinio el Viejo también nos dice que los esenios viven en la costa noroccidental del Mar Muerto, sine ulla femina, omni venere abdicata («sin ninguna mujer porque han renunciado a toda forma de actividad sexual») ${ }^{17}$. Si, de momento, aceptamos que los esenios eran idénticos, bien parcial o totalmente, a los habitantes del asentamiento de Qumran y los poseedores de los Manuscritos del Mar Muerto ${ }^{18}$, lo que encontramos no es sólo una llamativa confirmación de

${ }^{14}$ Todo este material es fácilmente accesible en A. ADAM y Chr. BURCHARD, Antike Berichte über die Essener ( $2^{\mathrm{a}}$ ed. Berlin 1972); la cita de Filón se encuentra en pág. 7.

${ }^{15}$ Véase, p.e., W. BAUER, «Essener», en Aufsätze und Kleine Schriften (Tübingen 1967) págs. 1-60.

${ }^{16}$ Véase, para lo que sigue, inter multos alios, J. M. BAUMGARTEN, «Celibacy», en Encyclopedia of the Dead Sea Scrolls, eds. L. H. SCHIFFMAN y J. C. VAN DER KAM, vol. 1 (Oxford 2000) págs. 122-125.

${ }^{17}$ Naturalis historia 5:15, 73.

${ }^{18}$ Esta cuestión tan debatida no puede discutirse aquí; véase el reciente artículo de T. S. BEALL, «Essenes», en Encyclopedia of the Dead Sea Scrolls, vol. 1, págs. 262-269. 
lo que Filón y Josefo dicen, sino que también tenemos buenas razones para consultar los Manuscritos del Mar Muerto sobre esta cuestión. Lo que encontramos es lo siguiente.

Incluso aunque la Regla de la Comunidad de Qumran (1QS) no diga en ningún lugar que el celibato es obligatorio para sus miembros, la comunidad de la que trata el documento parece ser una compuesta exclusivamente por un grupo de varones; las mujeres y los niños nunca se mencionan. En el conocido como Documento de Damasco $(C D)$, sin embargo, vemos que se presupone la presencia en la comunidad de mujeres casadas y de hijos, e incluso se dan normas para el matrimonio. Los expertos han sugerido que $C D$ es un documento que sufrió una serie de adaptaciones a lo largo del tiempo, y que las referencias a los matrimonios representan estratos posteriores que fueron añadidos para permitir que las personas casadas formaran parte de la comunidad esenia, pero esto siguen siendo conjeturas ${ }^{19}$. La Regla de la Congregación (1Q28a) simplemente da unas normas para el matrimonio. La situación se complica más por el hecho de que en los enterramientos que rodean Qumran predominan los esqueletos masculinos (circa $90 \%{ }^{20}$. Además, sólo se ha excavado un número de tumbas muy limitado. Sea como fuere, lo que está claro es que, exactamente como dice Josefo, había dos grupos dentro del movimiento esenio, uno estrictamente célibe, otro que no lo era. Lo que no sabemos es si estos dos grupos existieron simultáneamente o si se produjo un desarrollo desde un movimiento estrictamente célibe hacia una situación en la que, de forma gradual, se fueron tolerando, o aceptando, las parejas casadas. Lo que sigue siendo un hecho establecido es que hubo miembros del movimiento de Qumran y (o) esenio, que siguieron célibes por principio. Su conciencia de la necesidad de mantenerse en un estado continuo de preparación para la guerra escatológica (véase Megillat Milchama=1QM y 4Q491-496) puede haberles llevado de forma bastante natural y sencilla a una aceptación permanente de las reglas de la abstinencia sexual en una situación bélica, como ya se había formulado en la Biblia (Deut. 23:9-14). También la idea de que la comunidad de Qumran constituía un

19 J. M. BAumgarten, «Damascus Document», en Encyclopedia of the Dead Sea Scrolls, vol. 1, págs. 166-170.

20 Sobre estas tumbas, véase H. Stegemann, Die Essener, Qumran, Johannes der Täufer und Jesus (Freiburg 1993) págs. 72-74. 
templo espiritual, morada de Dios, ciertamente contribuyó a una fuerte convicción sobre la necesidad de mantenerse en un estado de constante pureza ritual ${ }^{21}$. Si tenemos en cuenta que en el libro del Exodo 19:15 se pide al pueblo de Israel que se abstenga de actividades sexuales con el fin de prepararse para recibir la revelación en el monte Sinaí, no es difícil imaginar que, en un grupo en el que muchos de sus miembros estaban probablemente en una expectación constante de nuevas revelaciones divinas, hubiera un fuerte deseo de permanecer en el estado de pureza ritual requerida para tales revelaciones ${ }^{22}$.

Esto nos lleva a un tema interesante que sólo podemos tratar de pasada, el de la abstinencia sexual de Moisés desde el momento de la revelación de la Ley en el monte Sinaí, o desde la auto-revelación divina en la zarza ardiente. La primera vez que se encuentra este tema es en la primera mitad del siglo I d.C., en la Vita Mosis (2:68-69), donde Filón dice que Moisés, para poder recibir las revelaciones de Dios, tenía que estar puro y, por tanto, tenía que abstenerse de todo contacto con mujeres. Dado que Moisés quería hallarse en un permanente estado de preparación para recibir la palabra divina -dice Filón-, desde el momento en que recibió la llamada permaneció libre de impureza por relaciones sexuales. Este tema se repite en la literatura rabínica ${ }^{23}$. Leemos en Sifre, el midrash rabínico, en Num.12:1-2 (\$\$99-100), que Miriam habló con Aaron sobre Moisés porque se había fijado en que la mujer etíope de éste había dejado de embellecer su rostro. Cuando le preguntó cuál era la razón, la mujer le había contestado que Moisés había dejado de interesarse por ella como mujer, por lo que Miriam llegó a la conclusión de que Moisés estaba descuidando el peru u-revu del Gen.1:28. En consecuencia, queda planteada la cuestión de cómo fue posible que los patriarcas, que también recibían continuamente mensajes de Dios, no se desentendieran de sus obligaciones maritales (una pregunta que sigue sin ser contestada). En otro midrash, Exod. Rabba 46:3, se dice que Moisés

\footnotetext{
21 Véase F. García Martinez y J. Trebolle Barrera, Los hombres de Qumrán. Literatura, estructura social y concepciones religiosas (Madrid 1993) págs. 165-186.

${ }^{22}$ Véase O. BETZ, Offenbarung und Schriftforschung in der Qumransekte (Tübingen 1960). Según Hechos 21:9, Felipe tenía cuatro hijas que eran vírgenes y (¿por esta razón?) estaban dotadas de dones proféticos.

${ }_{23}$ Véase BOyARIN, Carnal Israel, págs. 159-165.
} 
pensó: «Si se nos dijo que no nos acercásemos a una mujer en el Monte Sinaí, que fue santificado en una única ocasión por la revelación de la Torá [Ex. 19:15], con mayor razón debería yo, a quien Él habla todo el tiempo, alejarme de mi mujer». El constante celibato de Moisés es rubricado en otros pasajes aunque, a la vez, se insiste en que, a este respecto, Moisés no es en absoluto un modelo a seguir por todo israelita, ya que en los círculos rabínicos el ideal de fundar una familia estaba definitivamente asentado ${ }^{24}$. Incluso así nos encontramos con un tema importante: el celibato como fruto de la voluntad de una persona de permanecer en un estado ininterrumpido de pureza ritual con el fin de estar preparado para recibir un mensaje de Dios en cualquier momento. La conciencia de ser un profeta, o un miembro de una comunidad profética, llamada a estar abierta a la comunicación divina, pudo haber desempeñado un importante papel a la hora de llevar a muchas de las personas a las que nos estamos refiriendo aquí a abstenerse de toda actividad sexual.

No es improblable que sea a esta luz como hayamos de ver las vidas de dos importantes profetas judíos de la primera mitad del siglo I d.C., Juan Bautista y Jesús de Nazaret. En el Nuevo Testamento, los Evangelios nos presentan una descripción de Juan -su vida en el desierto, sus vestidos y alimentos (Mc. 1:4-6 y paralelos)- que recuerda enormemente a lo poco que, por Josefo, sabemos de Banus ${ }^{25}$. Aunque no sabemos cuál era el mensaje de Banus, está claro que Juan era un profeta escatológico. Su misión era de una gran urgencia: «iYa está el hacha puesta en la raíz de los árboles!» (Mat. 3:10 // Luc.3:9). Alguien que vive con la firme convicción de que el fin de los tiempos está próximo y cuya tarea es hacer una ultima llamada al pueblo judío para que se arrepienta y se convierta, ciertamente tiene unas prioridades que no son las de crear una familia, con todo lo que ello conlleva. Pablo, también, entendió muy bien esto: aconseja, en 1 Cor. 7:25-40, sobre si casarse o no hacerlo, todo ello motivado por la conciencia de que «el tiempo es corto» (v.29). En semejante situación es mejor permanecer soltero, porque la única cosa esencial y necesaria es «el trato asiduo con el Señor, sin división» (v.35). Es claro que uno

\footnotetext{
${ }^{24}$ Véase también Targumim en Num. 12:1-2; b. Shabbat $87^{a}$; Deut. Rabba 11:10; Avoth de-Rabbi Nathan (rec. A) 2:3; Mekhilta, Yithro Bachodesh 3.

${ }^{25}$ También en J. E. TAYLOR, The Immerser. John the Baptist within Second Temple Judaism (Grand Rapids 1997) págs. 32-42.
} 
no engendra hijos cuando el día del Juicio Final está a punto de llegar. En otras palabras, la urgencia escatológica es -aparte de la disposición a recibir las revelaciones de Dios en cualquier momento- un segundo motivo para trasladar el primer mandamiento de la Biblia (peru e-revu) a un segundo lugar. "Das Gebot der Stunde» tiene más peso que «das Gebot der ersten Stunde» en Gen.1.

Es aquí en donde, sin duda, hemos de mirar hacia el trasfondo del celibato de Jesús de Nazaret ${ }^{26}$. Es cierto que, por un lado, Jesús parece establecer un contraste entre sí mismo y su predecesor y maestro ascético Juan Bautista (que ayunaba mientras Jesús comía y bebía: Luc. 5:33-35, 7:33-35 y paralelos), pero, por otro lado, en lo que se refiere al matrimonio, están muy cerca el uno del otro. A pesar de las claras diferencias, ambos reciben al final la misma llamada, que han de cumplir por orden de Dios en favor de su pueblo. El matrimonio es incompatible con esta dedicación total. Tampoco es completamente imposible que los discípulos de Jesús fueran, también, hombres que habían dejado su vida de matrimonio. Es con este fondo como pueden cobrar sentido los comentarios de Jesús sobre la recompensa que los discípulos recibirían por haber abandonado, por amor al reino de Dios, casa, parientes, mujer e hijos, en respuesta a la afirmación, hecha por los discípulos, de que habían abandonado todo por seguirle ${ }^{27}$. La afirmación, en Mat.19:12, sobre aquellos que se habían hecho a sí mismos eunucos por amor al reino de los cielos pertenece, sin duda, no sólo a sus discípulos, sino también al mismo Jesús. El reino de Dios puede pedir renuncia a la vida sexual ${ }^{28}$. La libre asociación de Jesús con las mujeres -algunas de ellas de mala reputación- es evidente que no debe entenderse como contradictoria con esto. En

\footnotetext{
${ }^{26}$ Los muchos libros populares en los que se presenta a Jesús como casado con María Magdalena no tienen otro destino que la papelera. Para una exposición equilibrada de las pruebas y de la discusión moderna, véase el capítulo «¿Estaba casado Jesús?», en J. P. MEIER, A Marginal Jew. Rethinking the Historical Jesús, vol. 1 (New York 1991) págs. 332-345 (con notas en págs. 363-371), donde Meier también hace una breve, pero buena, presentación sobre las pruebas del celibato judío (págs. 336-339). Existe traducción española: Un judio marginal: nueva visión del Jesús histórico (Estella 1999).

${ }^{27}$ Véase, p.e., Mc. 10:29 // Mat. 19:29 // Luc. 18:29.

28 Véase W. D. Davies y D. C. Allison, A Critical and Exegetical Commentary on the Gospel according to Saint Matthew, vol. 3 (Edinburgh 1997) págs. 21-25. Véase también P. FredriksEn, Jesus of Nazareth, King of the Jews (New York 1999) págs. 98-110.
} 
el caso de Jesús hay otro factor que también interviene, concretamente su actitud fuertemente condenatoria hacia el deseo sexual en general ${ }^{29}$. Pasajes del Sermón de la Montaña -como aquel en que Jesús condena a quien mira a una mujer deseándola, como equivalente a haber cometido adulterio (Mt. 5:28), y los dichos que siguen sobre arrancarse los ojos y cortarse la mano (y en Mc. 9:45 también cortarse el pie ${ }^{30}$ )- indican claramente que los deseos sexuales han de ser erradicados por completo si uno quiere entrar en el reino de Dios (cf. Col. 3:5-6). La vida ideal, en adelante, de acuerdo con Jesús, es completamente asexual (Mc. 12:25: «Porque cuando resuciten de entre los muertos, no se casarán ni serán dados en matrimonio, pero serán como ángeles en el cielo»). Es posible anticiparse a esa bios angelikos ('vida como un ángel') desde el momento presente ${ }^{31}$.

Probablemente Jesús era indiferente al sexo, o quizás incluso lo viera negativamente. A este respecto no era en absoluto excepcional como figura profética, porque en los movimientos proféticos con frecuencia nos encontramos con que los modos fuertemente ascéticos suelen ir de la mano de un entusiasmo mesiánico-escatológico ${ }^{32}$. Cesar de tener relaciones sexuales es un modo de detener el mundo ${ }^{33}$. Pero esto no es todo. También se ha de tener en cuenta que en el tiempo de Jesús, después de tres siglos y medio de exposición a la cultura griega, los ideales ascéticos de los círculos filosóficos y religiosos de esta cultura habían penetrado lenta, pero firmemente, en el judaísmo palestino, y los judíos se habían apropiado de ellos ${ }^{34}$. Esto a veces tuvo la paradójica consecuencia de que, como dice Daniel Boyarin en su libro sobre Pablo, «mu-

\footnotetext{
29 Véase, para lo que sigue, D. C. Allison, Jesus of Nazareth. Millenarian Prophet (Minneapolis 1998) págs. 175-182.

30 'Pies' puede entenderse eufemísticamente como 'genitales'; véase W. DEMING, «Mark 9.42-10:12, Matthew 5:27-32 y B. Nid. 13 b: A First Century Discussion of Male Sexuality», New Testament Studies 36 (1990) págs. 130-141: pág. 134.

${ }^{31}$ Este es también el sentido del agraphon que se encuentra en 2 Clemens 12 (con un paralelo en Ev. Thomae 22), que no puede descartarse como del propio Jesús; véase T. BAARDA, "2 Clement 12 and the Sayings of Jesus», en Early Transmission of Words of Jesus (Amsterdam 1983) págs. 261-288.

32 Para ejemplos véase Allison, Jesus of Nazareth, págs. 194-196.

33 ALLison, Jesus of Nazareth, pág. 197.

${ }^{34}$ Véase, sobre este proceso, M. Hengel, Judentum und Hellenismus. Studien zu ihrer Begegnung unter besonderer Berïcksichtigung Palästinas bis zur Mitte des 2. Jhs. v. Chr. (Tübingen 1969) [= Judaism and Hellenism, London 1974].
} 
chos judíos del siglo I tenían el sentimiento de que Dios les había ordenado hacer lo que el propio Dios consideraba pecaminoso» ${ }^{35}$.

Filón es un buen ejemplo de esta «tensión ascética» en el judaísmo ${ }^{36}$. En su visión platónico-dualista, al verdadero creyente no le queda sino tratar de lograr una sola cosa, es decir, que el alma se libere de sus ataduras con el mundo material e intente alcanzar un estado de inmortalidad inmaterial. El mayor obstáculo en este proceso es el cuerpo con sus deseos carnales. La vida es, por tanto, una batalla constante con esos deseos, una lucha en la que la ayuda de Dios (inter alia bajo la forma de los mandamientos) es indispensable. Como acabamos de decir, Filón ve a Moisés, su gran y ejemplar héroe, como célibe, pero su ideal aparece mucho más claramente en su descripción del grupo de los Therapeutae, en un tratado con el expresivo título de De vita contemplativa. En él describe a un grupo de hombres y mujeres judíos que llevan una vida estrictamente célibe en un monasterio situado en una colina a las afueras de Alejandría, a orillas del lago Mareotis. Los Therapeutae están por completo dedicados al estudio de las Escrituras, a la oración, el canto y la contemplación; durante la semana cada uno de ellos se halla en la estricta soledad de su propia celda, pero se reúnen durante el sabat, en una celebración común en la cual los hombres y las mujeres están separados por una pared medianera tan alta como un hombre, de modo que no puedan verse entre sí. Viven una vida completamente sobria y, por esta razón, el ayuno es una parte integral de su estilo de vida. Nadie se extrañará de que varios estudiosos hayan sostenido en el pasado que este tratado no podía haber sido escrito por Filón, sino que debía pertenecer a un autor cristiano del siglo IV ${ }^{37}$. No es extraño tampoco que Eusebio de Cesarea califique como cristianos a los Therapeutae (Hist. eccl. 2:17). Las similitudes entre lo que sucedía en su propio tiempo en el naciente movimiento de los eremitas y los monjes del desierto eran demasiado llamativas como para admitir otra conclusión. Pero en la actualidad sabemos con

\footnotetext{
35 D. BoyArin, A Radical Jew. Paul and the Politics of Identity (Berkeley 1994) pág. 159.

36 Véase, p.e., D. Winston, «Philo and the Contemplative Life», en Jewish Spirituality, págs. 198-231; I. HEIEMANN, Philons griechische und jüdische Bildung (Hildesheim 1973 [Breslau 1932]) págs. 261-273.

37 Véase S. SAndmel, Philo of Alexandria (New York - Oxford 1979) pág. 35.
} 
seguridad que De vita contemplativa es realmente un texto de un filósofo judío, Filón, en el que se describe un movimiento monástico judío. Es cierto que los Therapeutae se muestran, sospechosamente, como personificaciones de los propios ideales de Filón, y por esta razón se ha sugerido que lo que Filón nos presenta es una descripción de una situación completamente ahistórica en la que los Therapeutae tienen que ser vistos como un modelo ideal. «Philo's De Vita Comtemplativa as a Philosopher's Dream» es el comprensible título de un estudio reciente sobre dicho tratado ${ }^{38}$. Pero no debemos olvidar que la renuncia al matrimonio por amor del estudio de las Sagradas Escrituras desempeñaba un papel central en el caso de Shim'on ben Azai, que ciertamente es un personaje histórico, y cuyo celibato no puede ser puesto en duda. Ya veamos la presentación de los Therapeutae hecha por Filón como una utopia, ya la veamos como una descripción idealizada de un grupo judío muy especial, lo que en cualquier caso queda es el hecho de que Filón sintió que podía presentar una vida ascética, sin matrimonio, como un ideal judío. Gen. 1:28 apenas representa ningún papel en el trabajo exegético de Filón, aunque en ningún lado afirme que es mejor no casarse porque el sexo no es bueno para la vida espiritual. Es posible que Filón fuera consciente del hecho de que su propia Sagrada Escritura parecía abrigar ideales que no eran los suyos, pero en tales casos era siempre Platón quien se lleva la palma, no Moisés.

Sin embargo, no fue Filón quien influyó en el judaísmo formativo, sino los rabinos. Estos últimos formularon reglas muy estrictas en lo relacionado con el cumplimiento de los deberes maritales -y afanarse por la lujuria o el placer no formaba parte de las mismas, ¡al contrario! ${ }^{39}-$, pero se tomaron el mandamiento de

\footnotetext{
${ }^{38}$ T. ENGBERG-PEDERSEN, Journal for the Study of Judaism 30 (1999) págs. 40-64.

39 Véase, p.e., D. BOYARIN, Carnal Israel: Reading Sex in Talmudic Culture (Berkeley 1993) págs. 61-76 et passim. Se debe recordar que los rabinos veían que la fuerza motriz que se encontraba tras la sexualidad era la «inclinación al mal» (yetser ha--ra); véase P. W. VAN DER HORST, «Evil Inclination», en Dictionary of Deities and Demons in the Bible, eds. K. VAN DER TOORNS, B. BECKING y P. W. VAN DER HORST, (2a ed. Leiden - Grand Rapids 1999) págs. 317-319. Para algunos ejemplos paganos de advertencias contra la búsqueda del placer en las relaciones sexuales, véase D. C. Allison, «Divorce, Celibacy, and Joseph (Matthew 1:18-25 and 19:1-12)», Journal for the Study of the New Testament 49 (1993) págs. 3-10: págs. 7-9.
} 
Gen.1:28 mucho más en serio que sus correligionarios ascéticos, como Filón. Nos podemos preguntar por qué los ideales de Filón no influyeron en el judaísmo, sino en el cristianismo. Es difícil tener cualquier tipo de certeza sobre esta cuestión, pero se puede conjeturar que esta forma de ascetismo no pudo hacerse más fuerte dentro del judaísmo debido, precisamente, a la glorificación de este ideal en la cristiandad. El antagonismo entre las dos religiones se había hecho demasiado grande como para hacer atractivo para una de ellas lo que era tenido como un ideal por la otra.

Hemos visto que la abstinencia sexual permanente se dio en el judaísmo temprano y que tuvo una diversidad de raíces. Una visión dualista del hombre y su mundo, influida por la filosofía griega, pudo haber sido un factor importante en el caso de Filón y, quizá, de los esenios. Una poderosa urgencia escatológica desempeñó, claramente, un papel en otros casos (Qumran, Juan Bautista, Jesús, quizá Banus). También la conciencia de ser el templo de Dios, o un ejército que se prepara para la guerra santa final, pudieron haber llevado a la abstinencia sexual (Manuscritos del Mar Muerto). Así mismo, la total devoción al estudio de la Torá pudo llevar a los individuos a renunciar a la actividad sexual (Ben Azai, los Therapeutae). En ningún caso puede explicarse el fenómeno por una sola causa. Lo que sí está claro es que, cuando nos encontramos en el Nuevo Testamento con los primeros impulsos de lo que más tarde acabaría por difundirse como el ideal cristiano del celibato (¡voluntario!), estamos, en el cristianismo temprano, ante algo que puede ser explicado sobre el trasfondo del judaísmo contemporáneo ${ }^{40}$. Esto no niega en absoluto que los ideales griegos hubieran penetrado en la primera cristiandad a través de un judaísmo (helenizado) ${ }^{41}$. No obstante, la visión tradicional de que el celibato es una innovación cristiana, resultado de una influencia exclusivamente griega, ha de ser revisada.

\footnotetext{
${ }^{40}$ Véase, así mismo, J. E. GOEHRING, «Asceticism», en Encyclopedia of Early Christianity, ed. E. Ferguson (2 $2^{\mathrm{a}}$ ed. New York - London 1998) págs. 127-130.

${ }^{41}$ Incluso en las primeras fuentes rabínicas se encuentran ideales típicamente greco-romanos en lo que respecta al comportamiento sexual: véase M. L. SATLOW, "Rhetoric and Assumptions: Romans and Rabbis on Sex», en Jews in a GraecoRoman World, ed. M. Goodman (Oxford 1998) págs. 135-144.
} 


\section{RESUMEN}

Aunque el primer mandamiento de la Biblia, «Creced y multiplicaos» (Gen. 1:28), no propiciara el desarrollo del ascetismo sexual en el judaísmo antiguo, hay claros testimonios de personas y grupos que eligen el celibato voluntario como una forma de vida judía. En este artículo se examinan las pruebas relativas a los esenios, los Therapeutae, Banus, Shim'on ben Azzai, Juan el Bautista y Jesús de Nazaret, así como sus consecuencias en el desarrollo del celibato en el cristianismo primitivo.

\section{SUMMARY}

Even though the first commandment in the Bible, «Be fruitful and multiply» (Gen. 1:28), countered the development of sexual asceticism in early Judaism, yet there is clear evidence of persons and groups choosing voluntary celibacy as a Jewish way of life. The evidence concerning the Essenes, the Therapeutae, Banus, Shim'on ben Azzai, John the Baptist and Jesus of Nazareth is discussed in this article as are the consequences for the rise of celibacy in early Christianity. 